\title{
The Interdependence of Research Funding Concentration, Policy Priorities, and Problem Choice
}

\author{
Emil Bargmann Madsen*
}

September 4, 2020

\begin{abstract}
The prioritisation of research funding towards a small elite of researchers and research topics of "strategic" importance are becoming a norm across national research systems. Researchers are increasingly worried that such steering hampers the diversity of scientific approaches and problems addressed. However, the effects of increased steering of who and what receives research funds are not well known. I use evidence from 65,000 research grants awarded by seven research councils in the United Kingdom and fifteen Danish research funders to investigate how strong funding concentration and thematic targeting leads to less topical diversity. Researchers in the very top of the funding distribution primarily investigate topics and disciplines with the most funding success, and research output form targeted funding schemes overlaps with that from investigatorled grants. Moreover, priorities from private funders line up with the type of research funded by public research councils. The findings highlight how steering through funding decisions can multiply across the scientific system and exacerbate existing funding concentration.
\end{abstract}

\section{Introduction}

Over the course of the past decade, the institutional structure of research funding have changed profoundly. This change have been especially visible in two areas: First, more academic research have become tangent on researchers' ability to secure funds and resources from sponsors external to their research organisation, including both public, private, and non-profit research funders (Whitley et al., 2018, p. 110). Combined with an increasing degree of competition for these funds and low success rates, funding is increasingly concentrated in the hands

*Danish Centre for Studies in Research and Research Policy, Department of Political Science, Aarhus University. Contact: ebm@ps.au.dk 
of a small elite of principal investigators and institutions (Berg, 2012; Bol et al., 2018; Katz \& Matter, 2019; Ma et al., 2015; Mongeon et al., 2016).

Second, the greater reliance on competitively- and externally evaluated grants have propelled funding decisions and priorities to the forefront of research policy tools for governments and other funders alike to utilise. This takes place in a direct way, as funders have sought to steer research in particular directions by allocating grants towards favoured research areas, problems, and approaches (Gläser \& Laudel, 2016; Gläser \& Velarde, 2018). Private funders, such as pharmaceutical or biotech companies, operate on a strict market-based logic, investing in research problems with a profitable potential (Jones \& Wilsdon, 2018; Yegros-Yegros et al., 2020). Even if these firms have diversified their research portfolio (Rafols et al., 2014, p. 27), biomedical research priorities remain tethered to the market for treatments rather than societal needs (Evans et al., 2014; Yao et al., 2015). Governments and public funding bodies have, on the other hand, attempted to ensure that such needs are prioritised in funded research by targeting areas and problems deemed "societally relevant". This has been the case in the United Kingdom, where research councils have adapted to new government priorities through greater activism in funding of selected areas (Kearnes \& Wienroth, 2011; Nature, 2003), or in Denmark where new strategic research councils and technology-oriented foundations were tasked with selecting projects that could foster innovation and solutions to society's problems (Aagaard, 2017).

While this direct steering of science towards certain research problems have garnered much interest, there is very little quantitative evidence of how funding concentration and policyinduced prioritisation in funding influences the direction research (Ciarli \& Ràfols, 2019; Gläser \& Laudel, 2016). How research content, including problem choice, is linked to changing funding practices is a pertinent question but still under-explored (Laudel \& Gläser, 2014, p. 1204; Whitley et al., 2018). The mechanisms governing how funding conditions influence both individual researchers' problem choice and the research system's wider topic and disciplinary diversity are likely many and interwoven. Without detailed knowledge of how scientists perceive and adapt to scarcity of research funds and the steering hereof, we cannot meaningfully separate the direct effects of funding arrangements from other incentives and constraints inheret to the scientific system.

I instead focus on the ways the growing concentration of research funding and funder prioritisation have facilitated greater steering in indirect or even unintended ways. I focus on two mechanisms related to the increased prioritisation of research funding. I ask:

1. Does stark concentration of resources for individuals coincide with a concentration within research problem/disciplinary choice?

The tendency for funders to increasingly prioritise few, "excellent", researchers are well established, and concerns for how it impacts the type of research conducted are prevalent. Fewer, and larger, grants could narrow the diversity of projects, induce more conservative assessment of grant proposals, and make applicants focus on problems with more certain outcomes (Bloch \& Sørensen, 2015, p. 36; Alberts et al., 2014; Fortin \& Currie, 2013). Moreover: 
2. How do prioritised, strategic, funding instruments in public research councils, and priorities from private funders impact "free"/investigator-initiated research?

Both prioritised public and private funding have been speculated to spill over into traditional grant applications by incentivising an overemphasis on certain problem areas (Bloch \& Sørensen, 2015, p. 36-37; Yegros-Yegros et al., 2020, p. 3).

To study this, I use information on approximately 65,000 research grants awarded by seven UK research councils, and fifteen public and private Danish research foundations. I focus on the strong concentration of funding for individuals and topics at the system-wide level, but also investigate what individual researchers choose to study, and how it relates to the amount of funding they receive and from what instruments and funders they recieve it.

I document that funding is indeed concentrated in both few individuals, research disciplines, and topics. To contextualise this finding, I also show that the well-funded principal investigators mainly engage in well-funded topics, and grantees seldom diversify their disciplinary and topical focus from grant to grant. Irregardless of the causal direction, funding seems to be biased towards more prestigious or popular topics. Furthermore, I show that both private and public research funders share priorities, and tend to fund the same topics the most. This prestige/popularity overlap could stem from the fact that the most successful grantees often receive funding from a more diverse set of funders.

These potential biases, and underlying perpetuating mechanisms, have major implications for research policy. It lends credence to already existing arguments in favour of dispersing research funding to obtain more diversity in research areas and approaches (see Aagaard et al., 2020, for a thorough discussion). Moreover, recent suggestions for implementing a modified lottery in grant competitions (Fang \& Casadevall, 2016) could potentially remedy the strong dual concentration for individual researchers and research problems. The paper makes several contributions to the brewing discussion of how to sponsor research, by illuminating the ways policy decisions about increased concentration and prioritisation of research funding may inadvertently exacerbate existing inequalities in the science system.

In the following, I revisit the existing evidence on the intersection of problem choice and funding concentration. Next, I lay out a rationale for how funding concentration on individual researcher may impact the spread of studied research problems at the systemic and individual level. I then describe the methodological base for the analysis of grants from Denmark and the United Kingdom, report on this, and finally discuss the implications of increased concentration for science policy.

\section{Interdependence in funding priorities}

The general scarcity of research funding budgets have long made it necessary for research funders to prioritise who they fund, and what type of research they support (Stewart, 1995; Waltman et al., 2019). Early accounts of how different lines of research are prioritised in the scientific system have generally framed it as a rational process where outcomes are selected in an optimal way given budget constraints (see Ciarli \& Ràfols, 2019, p. 952, for a discussion). 
The assumption has been that the research community is self-organising and self-regulating, and researchers will tend to allocate attention to the most important topics when left to their own devices (Polanyi, 1962; Sarewitz \& Pielke, 2007). In Polanyi's (1962) vision of science as a republic, scientists pursue problems and adjust their problem choice by taking into account what other researcher have worked or are working on. Prioritisation of different topics then emerges as guided by an invisible hand: Researchers self-coordinate, which leads to a joint result no individual intended. Accordingly, this self-coordinated focus on certain topics should ensure "the most efficient possible organization of science" (Polanyi, 1962, p. 54). As noted by Sarewitz and Pielke (2007), this is a very stylised view of how scientists choose what topics to work on, and few would argue that this extreme form of self-organisation takes place. However, many researchers and policy-makers may acknowledge that strategic investments in certain topics and disciplines are necessary, but societal needs are best addressed when researchers are relatively unrestricted in their pursuits (Sarewitz \& Pielke, 2007, p. 7).

Contrary to this, I will argue that prioritisation of different topics and disciplines through funding cannot be understood as a relatively free 'marketplace of ideas', where funding is allocated to areas proportional to their importance in the research community. Empirical studies have shown that scholarly attention and also funding is highly stratified, with few areas amassing disproportionate resources compared to the rest. A wealth of studies in biomedical science shows that research funding and publications are highly concentrated in few select areas. Grants from the US National Institutes of Health (NIH) tend to focus on a narrow set of diseases with around $40 \%$ of funding flowing to $10 \%$ of diseases, and funding distributions in one year often mirror previous years (e.g. Gillum et al., 2011; Gross et al., 1999; Yao et al., 2015). This would not be unexpected from the perspective of science as a self-coordinating system that I presented above. However, many studies show that there is often a gap between societal needs and research priorities. There is high relative investment in disease areas most likely to affect the populations in high income countries (Yegros-Yegros et al., 2020), investments often match interest by pharmaceutical companies (Evans et al., 2014), and already well-studied genes make up the bulk of genes studied in NIH-funded projects (Stoeger et al., 2018, p. 7). At the same time, research funding is increasingly concentrated in the hands of few researchers. Across many funders, grant amounts have increased, but are simultaneously awarded to a small selection of individuals (see e.g. Katz \& Matter, 2019; Ma et al., 2015; Madsen \& Aagaard, 2020; Mongeon et al., 2016). Often, around $20-25 \%$ of grantees recieve $75-80 \%$ of funding, all the while leading to decreasing marginal returns in terms of publications and other research output (Aagaard et al., 2020; Mongeon et al., 2016).

I present two perspectives on how the concentration of funding in individuals and topics interact through different prioritisation mechanisms. First, prioritisation can be aimed at how science is conducted (e.g in large or small teams) or who is involved (a small 'elite' of researchers or a broader growth layer). This type of prioritisation takes place as funders increase grant sizes, while success rates decline and grant money become increasingly concentrated. Second, prioritisation can happen through select emphasis. Here, priority is placed on the substantive content of scientific research by designating particular disciplines or research topics, on which privileged status is bestowed on. Such thematic priorities are the most common way of think- 
ing about research funding priorities (Hellström et al., 2017; Jones \& Wilsdon, 2018; Wallace \& Ràfols, 2018).

Below, I outline how these prioritisation mechanisms may impact the distribution of funding to different topics and disciplines in the aggregate, but also how it could affect the topic choice of individual researchers in a stratified manner. Where individual choices of research question affects the career trajectory of the researcher, scientists' combined choices affect the direction and efficiency of scientific discovery as a whole (Rzhetsky et al., 2015).

\subsection{Individual funding concentration and topic choice}

The connection between increasing concentration of funding and a lack of diversity in what topics and research areas are studied have received much attention and speculation. A high degree of systemic funding concentration is thought to inhibit risk taking in funding competitions. Funding a proposal can be thought of as an experiment with uncertain outcomes. When funding is concentrated on a small group it induces risk as the number of experiments are reduced (Bloch \& Sørensen, 2015; Fortin \& Currie, 2013). This can perhaps explain greater concentration of funding in few topics, as researchers choose topics with a proven track record, which in turn creates a more static and less diverse system (see Aagaard et al., 2020). This would explain why e.g. funded projects focus on already well-studied genes and molecular entities, and not those with more unknown functions (Edwards et al., 2011; Stoeger et al., 2018). However, without access to the inner thought process of researchers when applying to funding, we do not know whether this is the case.

Instead, we can think of the prioritisation of already well-funded researchers as bringing broader organisational issues, which inhibit the diversity of topics and research areas funded. Funding concentration disproportionally affects early-career researchers, who cannot compete with the publication record and resources of a well-funded senior elite (Aagaard et al., 2020; Peifer, 2017). Moreover, it contributes to the broader cumulative advantage of certain privileged individuals and research groups (Allison et al., 1982; Cole, 1970; Merton, 1968). Cumulative advantage in research funding signifies that individual researchers receive differential levels of funding depending on earlier funding, recognition, and type of work they have done. For example, scientists studying the least popular quintile of genes are around 12 percentage point less likely to win subsequent funding compared to scientist focusing on the most studied genes (Stoeger et al., 2018, fig. S10). So when grant sizes increases but success rates are low ${ }^{1}$, some successful research group have much better access to resources, which can be used in subsequent funding competitions. This can result in a continued stratified support for a narrow set of specialised research areas and groups working within particular fields. The possible cumulative advantage of well-funded grantees leads to a set of expectations about the funding distribution at the aggregate level and the level of the individual researcher.

\footnotetext{
${ }^{1}$ Between 2011 and 2019, success rates for obtaining a grant have fluctuated between 14 and $19 \%$ in the Independent Research Fund Denmark (which includes the five research councils). In UK research councils, the success rate in e.g. the Biotechnology and Biological Sciences Research Council saw a drop from 30 to $25 \%$.
} 
Aggregate funding distribution. If the distribution of funding to individuals and topics is interdependent, we should expect the cumulative distribution of funding to individual investigators to be considerably skewed, and the aggregate distribution of funded topics to be just as unequal. However, it is important to note that these systemic characteristics would not in itself suggest an interdependence of funding inequalities. The distribution of funding to topics could be unequal because all grantees focus on a smaller set of topics, in line with what would be expected from the self-coordination perspective. The interdependence should also be visible at the level of individual researchers.

Topic distributions across individual researchers. In order for cumulative advantage to drive the unequal distribution of funding to topics, I expect researchers with different levels of funding resources to investigate very different topics, and researchers in the top of the funding distributions should also focus on the topics with the most funding. Conversely, if the two distributions are independent, a skewed distribution of funding to topics will stem from researchers across the funding distribution researching similar topics.

\subsection{Thematic priorities, private funders, and spill-over of topic-choice}

The strong competition for funding, and its consequences for topic diversity, is a concentration mechanism internal to the scientific system. Greater competition and notions of what constitutes excellent types of research and researchers are likely to become internalised in funders' assessment criteria. The use of these criteria then signals to researchers, journals, and reviewers what topics are deemed prestigious (Madsen \& Aagaard, 2020, p. 17). However, a simultaneous development in the funding system have been the growing external steering of research through funding allocations to thematic priority areas and problems (Gläser \& Laudel, 2016, p. 126; Whitley et al., 2018, p. 110). This targeting of research funding has been driven by both public research funders and private companies, foundations, and charities. Crucially, targeted funding signals to the scientific community what areas and topics are important and where to expect future possibilities for funding. Targeted priorities are especially argued to solidify emerging fields such as nanotechnology or synthetic biology (Gläser \& Laudel, 2016, p. 127), but then continuing to skew research efforts through continued investment in these few strategic areas (Nature, 2003). Differences in assessment criteria can further amplify this skew. In the Biotechnology and Biological Sciences Research Council (BBSRC), "Excellence is the overriding criterion in the assessment of research grants (...)", but "(...) it is expected that competitive applications that address a responsive mode priority will have some advantage in competition." (BBSRC, 2020). In the Engineering and Physical Sciences Research Council (EPSRC) researchers have experienced much starker declines in success rates for investigator-initiated programmes than targeted programmes (Nature, 2003).

For private funders, the use of targeted priorities tend to be more "acceptable" for the research community. Many private funders operate within certain market demands and focus on research with a profitable potential (Sarewitz \& Pielke, 2007; Yegros-Yegros et al., 2020). However, because private funders in especially health and biomedical research have expanded their 
research investments this may affect publicly funded research too. When $60 \%$ of health research is rooted in the private sector, and many pharmaceutical companies spend more money on research than e.g. the Wellcome Trust (Røttingen et al., 2013), funding from industry will likely influence the topic selection of researchers even if their studies are based on public funding. Pharmaceutical research priorities then "spill-over" into public research priorities (YegrosYegros et al., 2020, p. 3; Evans et al., 2014), perhaps because some areas are easier to attain funding for. Targeting by public and private funders can also lead to a set of expectations about both aggregate funding distributions, and individual researchers' topic choices.

Aggregate funding overlap. Spill-over between targeting and private priorities to more "free" funding programmes and instruments will entail a positive relationship in the relative distribution of funding to different topics and disciplines between the programmes. Put plainly, those topics and disciplines in the top of the funding distribution in targeted or private funding programmes, should be similarly placed in funding programmes or organisations with less topical binding.

Targeted grants and topic choice. It is important to note that an overlap in themes funded by public vs. private funders or targted vs. non-targted funding programmes may suggest spillover, but not how these spill-overs manifest for individual researchers. Topic-choice is a complex task, and researchers likely select problems that strike a balance between their own interest, what the scientific community deem important, and what is expected by funders (YegrosYegros et al., 2020, p. 2). The impact of targeted funding on individual topic choice could play out in different ways. First, targeted funding opportunities could lead researchers in some areas to abandon or change their focus in order to match prioritised areas. Second, targeted programmes could instead privilege scientist already working in prioritised areas, giving them a competitive edge in other funding competitions. I return to all the outlined expectations in the analyses below.

\section{Data and methods}

\subsection{Data on research grants}

To identify how funders allocate resources to different research topics and areas, I rely on detailed data on both funded research grants and the output produced from these grants in the form of scholarly publications. The data on grants come from seven public research councils in the United Kingdom, and fifteen major research funders (incl. public and private) from Denmark. ${ }^{2}$ For the United Kingdom sample, I scraped all available information on funded research

\footnotetext{
${ }^{2}$ The funders are: The Carlsberg Foundation, the Independent Research Fund Denmark (DFF), the Danish National Research Foundation (DNRF), the Council for Technology and Innovation, the Strategic Research Council, the Advanced Technology Foundation, the European Research Council (ERC), the Danish Cancer Society, the Lundbeck Foundation, the Nordea Foundation, the Novo Nordisk Foundation, TrygFonden, the Ministry for
} 
Table 1: Summary of grant samples

\begin{tabular}{lcccccc}
\hline & & & \multicolumn{3}{c}{ Grant value $^{\mathrm{a}}$} \\
\cline { 5 - 7 } Sample & Years & No. of grants & No. of PIs & Mean & Median & Std. dev. \\
\hline Denmark & $2005-2016$ & 18,404 & 8,624 & $2,555,962$ & $984,191.0$ & $6,690,068$ \\
United Kingdom & $2005-2017$ & 41,416 & 22,290 & 434,732 & $239,807.2$ & $1,425,052$ \\
\hline
\end{tabular}

Note: ${ }^{\text {a }}$ Values are given in DKK and CPI-adjusted pounds respectively.

projects from the AHRC, BBSRC, EPSRC, ESRC, MRC, NERC, and STFC ${ }^{3}$ in the RCUK Gateway to Research Database from 2005 to 2017, in the BBSRCs advanced grant search, and the EPSRC Grants on the Web database. For the Danish sample, individual funders were contacted and asked to provide project-level data from 2004 to 2016. Table 1 provides an overview of the two samples of grants.

In both cases, the number of grants do not provide a full picture of all grants awarded by the United Kingdom research councils or Danish research funders in that period. For both samples, many projects are not strictly research-related or aimed at producing a traditional scientific output. I have excluded all grants aimed at training or education, and kept pure research grants and fellowships. Furthermore, all grants were linked to the primary principal investigator only, and grants with a running amount paid by year were centered on the first year the grant was received. For the United Kingdom sample, I rely on the the individual investigator ID provided by the research councils to disambiguate individual PIs. Grants where a PI could not be disambiguated where kept out of the analysis, yielding 41,416/53,095 $\approx 78 \%$ of the total number of grants. For the Danish sample, grant recipients were manually disambiguated and linked to grants. For common name combinations, the grant recipient was identified through a combination of data on their institutional affiliation and primary research area. If not successfully disambiguated through this, grantees were considered separate individuals. Consequently, the sample has less comprehensive coverage of strategic and innovation-related funding, as these types of grants are often awarded to a consortium of firms and research organisations with no single discernible PI. These limitations are important in qualifying the results presented below. Despite this, the samples cover larges parts of competitive funding awarded over a twelve year period, and while they may not accurately depict the absolute funding amounts, they provide important insights about the relative distribution of scarce resources in the research systems.

Higher Education and Research, the Velux Foundation, and the Villum Foundation

${ }^{3}$ Arts and Humanities Research Council (AHRC), Biotechnology and Biological Sciences Research Council (BBSRC), Engineering and Physical Sciences Research Council (EPSRC), Medical Research Council (MRC), Natural Environment Research Council (NERC), and the Science and Technology Facilities Council (STFC) 


\subsection{Categorising grants into topics and disciplines}

For both the United Kingdom research councils and the Danish research foundations, we lack a consistent system for classifying grants into research disciplines and research topics. First, while the UK grants are often classified from a standardised scheme by the grantee themselves, the classifications schemes differ across councils. Second, these classifications seem to work on very different levels of aggregation with the MRC using 26 areas for classification while e.g. the EPSRC uses over 200. Third, we cannot compare across the UK and Danish samples without establishing a common classification system.

To consistently classify grants, I use journal articles and reviews by the principal investigators of each funded project. This do entail some methodological assumptions, as I expect a principal investigator's publications to reveal the underlying mix of topics and disciplines of the grant. I also assume that the topic mix has not changed much from grant proposal to output. Using the publication output also means that I am assessing the type of research that a grant actually resulted in, instead of just its intended results. For grants in the UK sample, the Gateway to Research database provides DOIs of publications reported by the grantees themselves as being outcomes from their grants. I match these DOIs to records in the Centre for Science and Technology Studies' (CWTS, Leiden University) in-house version of Web of Science (WoS). For Danish grants, I combined automated string and manual matching, by comparing names, email addresses and institutional affiliations in WoS with the grant data. Here, I cannot discern what publications were actual outputs from a grant. Instead, I consider the publications 0-4 years after a grant was received. None of the samples were completely covered by the WoS data, but the matched grants account for around $70 \%$ of the combined funding amounts attributed to individual principal investigators. Table $\mathrm{S} 1$ in the supplementary material shows comparisons between the full samples and the WoS-matched samples.

Using the matched publications, I categorise grants into two different levels of aggregation, which merit further explanation. First, I situate each grant within a scientific topic. A topic can be thought of as the lowest level of aggregation in a hierarchical, content-focused, categorisation scheme. I understand a research topic as a cognitive and semantic space populated by a collection of documents focusing on a common problem area, and with a set of researchers engaged in this problem area. A topic is cognitive because researchers identify as parts of this topic and group text based on this, and semantic because documents and researchers share a common vocabulary and understanding of key terms within this topic. To identify the research topic of a grant, I use algorithmically delinated topic clusters. The clusters are created by the CWTS, and used in their Leiden Ranking of universities. ${ }^{4}$ The topic of a publication is based on the direct citation links between the publication and all other publications in the WoS. Each article constitutes a node in a network with direct citations between papers constituting an edge between a pair of nodes. A Smart Local Moving (SLM) algorithm (Waltman \& Van Eck, 2013) is applied for community detection and clustering into topics. Individual article nodes are moved into a partition and then aggregated so that each topic constitutes a node in an aggregated network. This procedure is outlined in (Waltman \& van Eck, 2012) and (Traag et al.,

\footnotetext{
${ }^{4}$ https://www.leidenranking.com/information/fields
} 
2019). In all categorisation of research topics, a crucial question is the choice of granularity or resolution, i.e to choose how many different topics to include. I use two levels of granularity: a set of 3999 micro level topics, and a set of 864 meso level topics. These topics are hierarchically nested such that each micro topic is assigned to one meso topic, and each meso topic comprises a set of related micro topics.

Second, I also place each grant in a set of 250 disciplines or specialities, based on the WoS Subject Classification. Disciplines are more aggregate categories of the scientific structure than topics, and denote a self-organised network of scientists working within the same topics, publishing in the same journals and readings/citing each others work (Sjögårde \& Ahlgren, 2020, p. 210). I use the original classification of papers into disciplines, where each journal is classified, and a paper receives the same classification as the journal it was published in. However, this poses a problem because not all papers within a journal necessarily address the same discipline. To mitigate this, I follow Milojević (2020), and categorise a paper based on the most frequent discipline present in a papers cited references. This also eliminate a categorisation of grants into the residual "Multidisciplinary Sciences"-category, as papers are assigned the most prevalent category from their references except for this.

These four types of topic and discipline categorisations are by no means "the correct" classification, but there are likely no "ground truths" about how to classify a set of documents. The four categorisations I use instead fit the analysis in this paper by providing both different levels of aggregation and highlighting consistencies and inconsistencies across them.

The final categorisation of grants, places each grant into a mix of topics (two levels of aggregation) and disciplines (two methods), by weighting a grant according to its mix of publications. For each grant, I weigh both the grant sum and the grant itself according to the proportion of

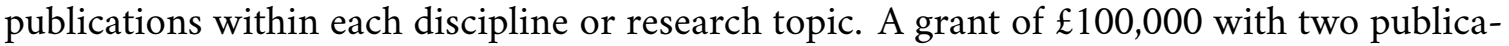
tions within cell biology (66.6\%) and two publications within virology (33.3\%) is divided so

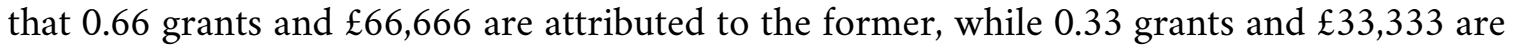
attributed to the latter.

\subsection{Case selection and data considerations}

I use data from both Danish research funders and United Kingdom research councils because each grant sample allows me to investigate different research questions. The data on Danish research grants cover a large section of the research funding system, and includes grants from private research foundations with ties to different industries (e.g. pharmaceutical companies, a brewery, and a window manufacturing company). This broad coverage makes it possible to compare how funders from both the public and private sector prioritise different research topics and disciplines, or the degree of overlap in their priorities. Furthermore, the inclusion of private funders, regular research councils, and strategic research councils provide a more encompassing view of the funding system and the distribution of resources across it.

The United Kingdom data does not support such an analysis, but carries other advantages. The data from the UK research councils can be directly linked to research publications produced within the scope of each individual grant. This makes it possible to separate which topics 
Funding concentration in Denmark and the United Kingdom
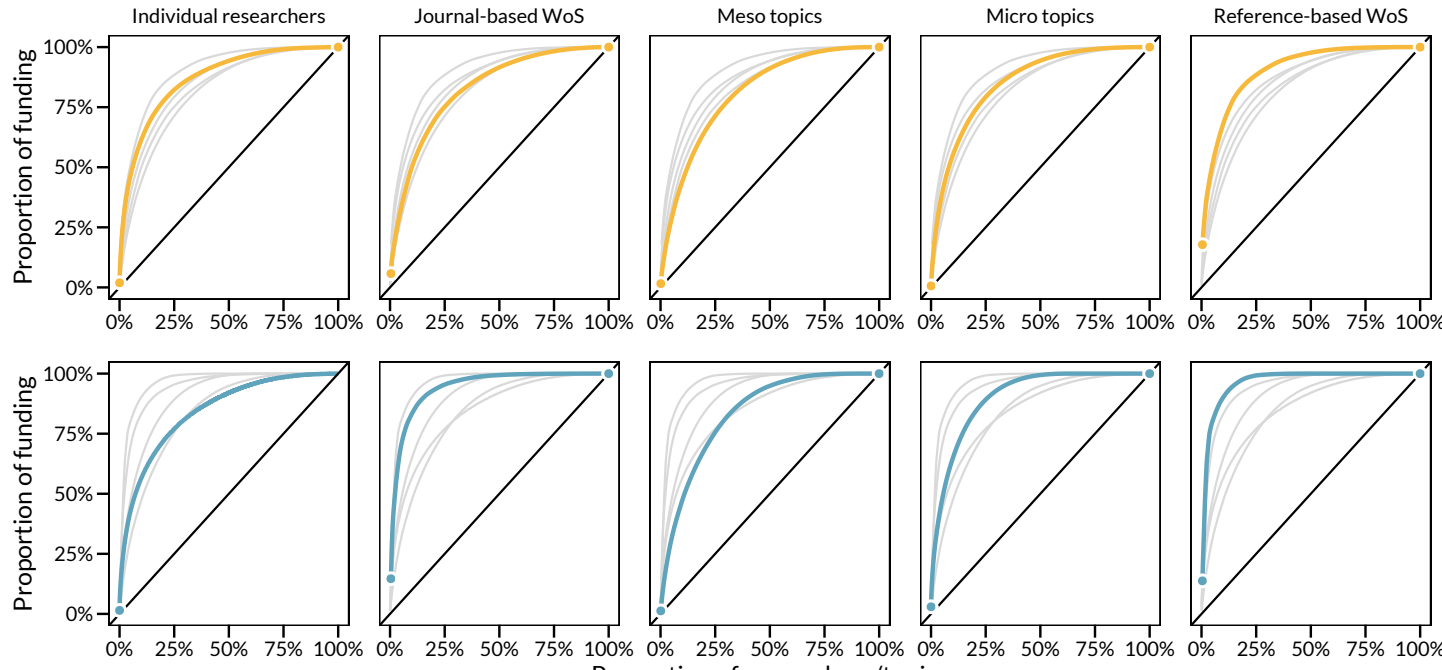

Proportion of researchers/topics

Figure 1: Funding concentration at the indvidual, topical, and disciplinary level.

and disciplines each grant engages in, and compare several grants awarded to one PI. The cases thus provide different opportunities, and I do not explicitly compare the cases in order to highlight any systematic and theoretically meaningful difference. Instead, the results show many commonalities between the cases, which may indicate some robustness of the findings.

\section{Results}

While much speculation have linked the increasing degree of funding concentration on a small group of "excellent" or "elite" researchers, and the tendency for funding to flow towards a narrow selection of research areas, disciplines, and topics, this interdependence is not well understood. Until recently, we knew very little about how research funders prioritise grant money, and how their selection criteria come to impact the type of research being pursued. Recent case studies in medicine (Yegros-Yegros et al., 2020), but also large-scale mappings of all areas (Waltman et al., 2019) show that funding is highly skewed towards certain disease areas and research topics. As outlined above, the missing piece of the puzzle is the underlying mechanisms driving these imbalances or concentration trends. Such mechanisms are likely many, and may combine in complex and unpredictable ways. Factors such as stratified publication incentives for different topics, commercial interests, and geographical specialization all seem to been important drivers (Ciarli \& Ràfols, 2019; Yegros-Yegros et al., 2020). To get an overview of the degree of funding concentration, Figure 1 shows the Lorenz curves for funding across both individual researchers and the four levels of research topics and disciplines.

Broadly speaking, there tends to be a strong concentration of funding across researchers, topics, and disciplines and in both Denmark and UK. For individual researchers, the amount of 
funding is distributed so that $25 \%$ of Danish grantees are awarded $82.2 \%$ of all funding while $25 \%$ of the UK grantees are awarded $77 \%$. This tendency for concentration is similarly strong across both micro- and meso-level topics. Across the 15 Danish research funders, $25 \%$ of micro topics amass around $79 \%$ of the total funding amounts in the years 2005-2016, while the concentration is somewhat reduced for the more aggregate meso-level with $71 \%$. For the UK, the degree of concentration is even stronger with a 25/76 and 25/89-division respectively. One explanation for the slight differences between Denmark and the UK could be the difference in funder composition. The Danish data are more encompassing and include grants from private research foundations. These grants may be directed at slightly different topics than what is funded by public research councils, as many of the Danish private foundations have more narrow scopes for their allocation defined in their charters. If we take a more aggregate look at the types of disciplines prioritised in the funding systems, we also find evidence for a very strong concentration trend. Using the regular WoS categorisation scheme, Danish funding is concentrated in a small handful of disciplines with $75 \%$ of funding being directed at $25 \%$, or 63 disciplines, while 63 disciplines amass $95 \%$ of funding in the UK. However, a lot of funding is directed towards "Multidisciplinary Sciences" because the original WoS categorisation bundles all multidisciplinary journals into one category. If we re-categorise these according to the most prevalent, non-multidisciplinary, category in the reference list we see an even stronger degree of concentration. For Denmark, the division is then 25/88 while the UK see an extreme 25/99 split.

Taken together, the distribution of funding amounts across individuals, topics, and disciplines show a strong stratification with few individuals and areas being prioritised, while the bulk remain scarcely funded. The simultaneous existence of skewed funding distributions indicate that at least some of the intuition from above could be true. Strong individual concentration could lead to topic concentration, or vice versa. It could also be that strong topic concentration is not due to few researchers getting a lot of funding for specific topics, but that all funded researchers engage in popular topics as a consequence of e.g. strong specialization, choosing topics based on opportunity, or survivor ships bias where only researchers engaged in popular topics stay in academia, get funding, etc. To more closely examine the interdependence of these funding distributions, we need to observe how individuals in different strata of the funding distribution choose topics.

\subsection{Topic and funding concentration at the researcher level}

So while the system-wide concentration of funding at both the individual and topical level is strong, a lack of diversity in who is funded does not automatically translate into less diversity in what is funded. If a wide selection of researchers engage in the same disciplines and topics, strong funding stratification is merely a sign of many grants (both small and large) flowing towards these areas and a relatively narrow focus from all grantees. This would create a positive self-reinforcing feedback for well-funded topics, but not necessarily a negative selfreinforcing feedback for low-funded topics. The collective topic choice could shift when new topics become popular or prestigious. Conversely, when a narrow elite of researchers engage 
Researcers working on a top funded topic in Denmark and the United Kingdom
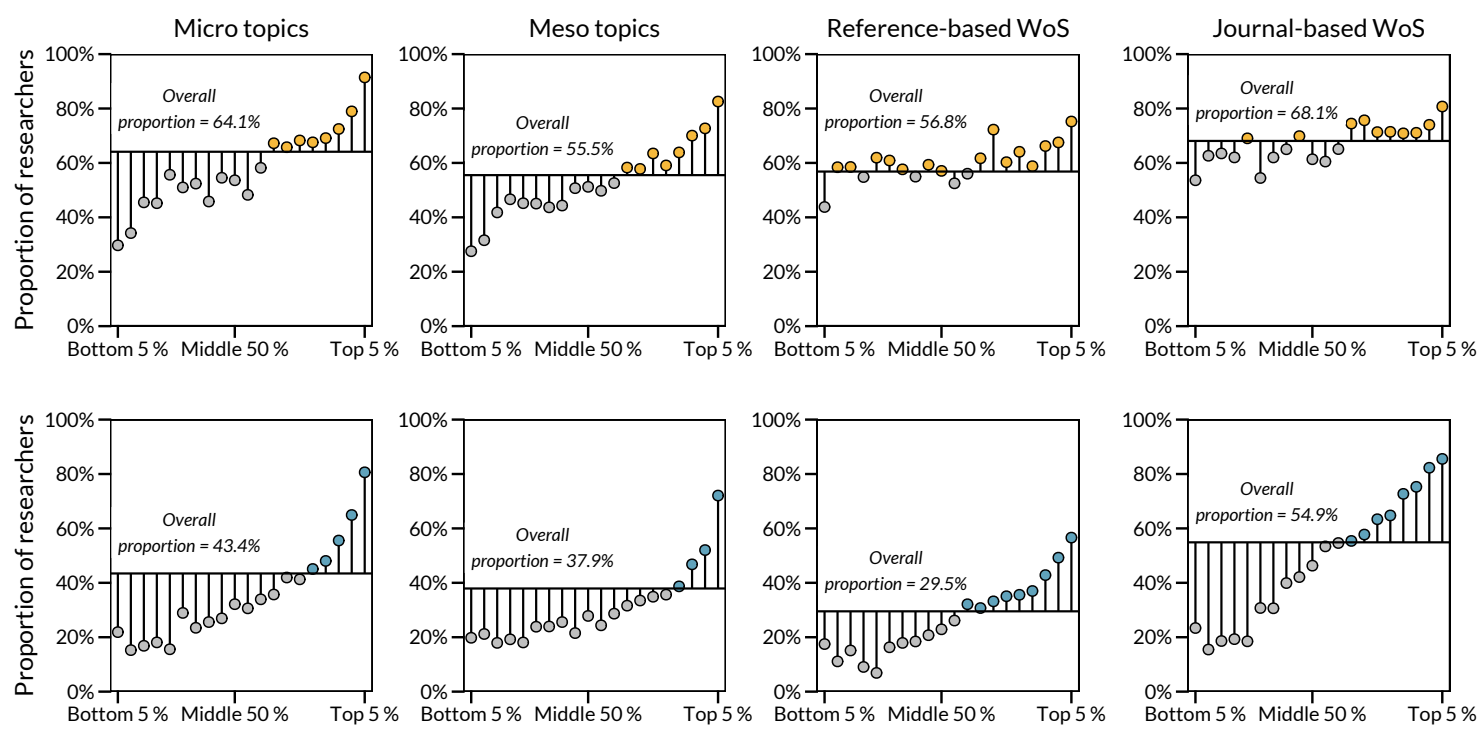

Figure 2: Individual funding concentration and propensity to work in top $5 \%$ funded research fields.

with certain topics in a select range of disciplines, and relatively poorer researchers engage in different topics, with less funding success, the interdependence creates negative spirals from some researchers and topics.

Figure 2 ranks each grant recipient into 20 percentile ranks according to the cumulative amount of funding awarded to that person. This gives us 20 equally sized groups of researchers from different parts of the funding distribution. Next, we can calculate the proportion of researchers in each percentile rank group (e.g. the top $5 \%$ funded researchers) who also work with a top $5 \%$ funded topic or discipline, and compare it to the proportion of researchers across all funding ranks working within a top $5 \%$ funded topic.

Across the Danish and UK case, we see a common pattern: More well-funded researchers tend to work within well-funded topics and disciplines, while researchers further down the funding distribution have lower propensity to engage in these. In the Danish case, researchers across the funding distribution tend to work in top funded topics and disciplines, with 55-68 $\%$ of grantees addressing a top $5 \%$ funded topic or discipline in some way. Looking at the disciplinary level, some differences across funding ranks exist but there seems to be very little structure to these differences. Generally, $60-80 \%$ of researchers within the top $50 \%$ of the funding distribution work in the most well-off disciplines, where this is just the case for around $40-60 \%$ in the bottom part. However, large differences emerge at the topical levels. For meso-level topics, over $80 \%$ of the top funded grantees work on the most funded topics, while less than $30 \%$ of the least funded grantees work with these. At the most fine-grained topic level this difference is even bigger (insert difference). There are many possible interpretations of these patterns. First, I seems that funded researchers in Denmark are fairly specialised with 
many grantees working within a narrow set of topics and disciplines. Second, the lack of stratification in disciplinary focus vs. the strong stratification in topic focus is interesting. One explanation can be that biomedicine and clinical medicine are particular areas of funding and specialisation in Denmark (see Madsen \& Aagaard, 2020, p. 12), but even within these areas a select group of problems and diseases are heavily funded. For example, diabetes mellitus drew approximately $11.8 \%$ of disease-realted research funding in the period under consideration (Madsen \& Aagaard, 2020, p. 13).

In the $\mathrm{UK}$, the pattern of stratification is quite similar in all conceptualisations. For microlevel topics, the difference between the top $5 \%$ and bottom $5 \%$ is almost 59 percentage points. In the top, over $80 \%$ of grantees work on top funded topics whereas only $22 \%$ of the bottom part of the funding distribution do the same. For meso-level topics, the difference is 52 percentage points, 50 percentage points for reference-based disciplines, and 62 percentage points for journal-based disciplines. In contrast to Denmark, the disciplinary and topical focus of UK grantees are less concentrated on heavily funded areas. The overall proportion of researchers working in these fields are around 30-55 \%, but also varies much more between the disciplinary and topical level. It is interesting to note the difference between the reference-based categories and the original WoS categorisation. For the former, only $30 \%$ of researchers focus on well-funded topics but the latter categorisation changes this to $55 \%$. A likely explanation is the heavy focus on prestigious, multidisciplinary, journals in many disciplines. When using the re-classification proposed by Milojević (2020), a grant's papers in Nature, Science, PNAS, etc. are redistributed to other disciplines.

Another contributing factor to the large degree of topical stratification can be that researchers tend to work on very related topics throughout their career. When the well-funded work on the most prioritised topics, and continue to do this, the funding concentration reproduces across time. Figure 3 compares the topical and disciplinary focus of a PIs first grant to all subsequent grants (for researchers with $>2$ grants). This is possible with the UK data, as we can specifically link each grant to their publication outcomes, and thus distinguish the topical focus of individual grants. The measure for similarity is computed as the cosine similarity between a vector of topics addressed in a PIs first recorded grant (A), and a vector of topics from grants acquired 1, 2, 3, ..., n years after this (B). Each cell of these vectors take on a value equal to the proportion of papers from a grant that addresses a certain topic, such that topics not addressed equals 0 , while topics adressed in a quarter of the publications equals 0.25 :

$$
\text { similarity }=\frac{\sum_{i=1}^{n} A_{i} B_{i}}{\sqrt{\sum_{i=1}^{n} A_{i}^{2}} \sqrt{\sum_{i=1}^{n} B_{i}^{2}}}
$$

where $A_{i}$ and $B_{i}$ are a topical or disciplinary proportion.

In the plot, a similarity score of 1 indicates a perfect overlap in topic or discipline profile between two grants, while 0 denote no overlap. Because the distribution of similarity scores are skewed towards 1, I show both the mean and median score. In general, a a PIs grant tends to be very similar in scope with mean and median similarity scores between 0.5 and 0.75 . Naturally, a researcher's grants tend to be more similar at the disciplinary level than the topical level, and the similarity decreases with the number of topics we use to categorise. So while early grants 


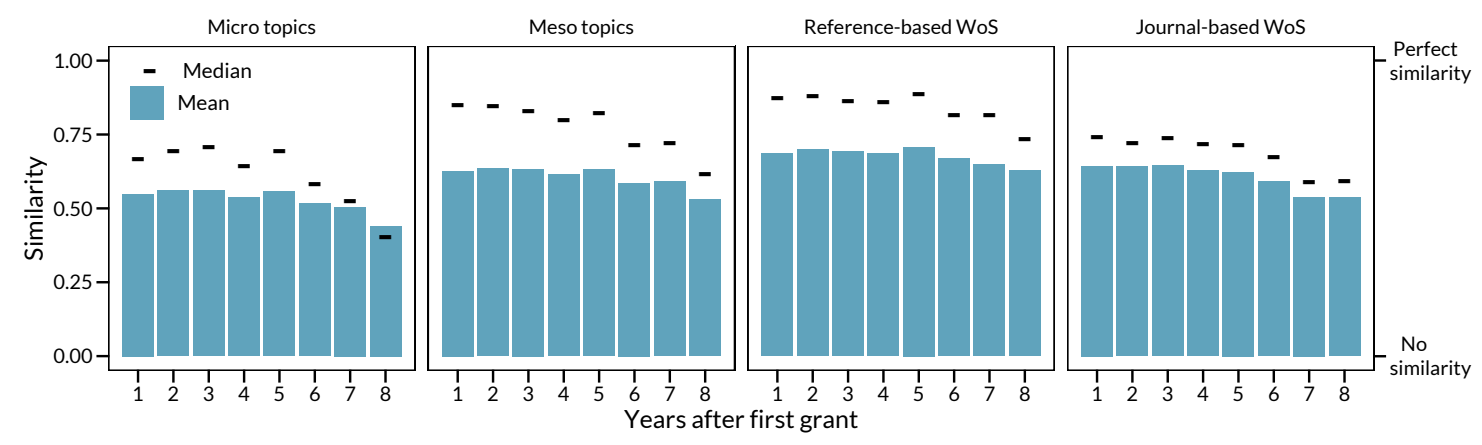

Figure 3: Topical similarity of grants across a PIs career in the United Kingdom.

seem very predictive of later topical focus, researcher do have room to shift topical focus within a broader set research agenda. Moreover we see a slight tendency for topic and disciplinary similarities to lessen as time goes by, indicating that researcher are branching out later in their careers. This largely corroborates findings from other analyses of topic switching behaviour (Horlings \& Gurney, 2013; Zeng et al., 2019). However, the similarity scores are only based on a sub-sample of researchers and may not be representative of the wider set of grantees.

In sum, the distribution of funding to topics, disciplines, and individual researchers are clearly linked with strong indications that highly funded researchers also research the most prioritised or popular topics. This stratification in topic focus across the funding distribution is likely one mechanism driving the huge inequalities in what type of research is funded in both Denmark and the United Kingdom. Furthermore, the strong path dependency of early projects could possibly contribute to a persistent strong concentration, as researchers across the funding distribution rarely change topic or disciplinary focus.

\subsection{Overlapping priorities in diverse funding landscapes}

The preceding analyses show how a prioritisation of funding towards a small segment of the researcher, or grantee, population may serve to increase the concentration of funding towards a handful of topics and disciplines. Another commonplace explanation of the interdependence of funding concentration for individuals and topics, are the specific topical priorities set by research funders. Recall that thematic priorities come in various implementations. First, public research funders have generally embraced the thought of earmarking funding to specific disciplines or topics deemed societally relevant, and have set aside funding instruments to encourage more research in these areas. Second, private funders have long focused on areas that are advantageous to their stated goals in charters or market strategies. Both types may lead to a spill-over of research priorities by incentivising research in specific research areas, and making it possible for already successful researchers to pull funding from many different sources.

Priorities in public funding. In figure 4, I aim to compare the relative priority or emphasis that strategic research councils or prioritised funding instruments put on certain topics, to 
Earmarked vs. investigator-led funding in Denmark and the United Kingdom
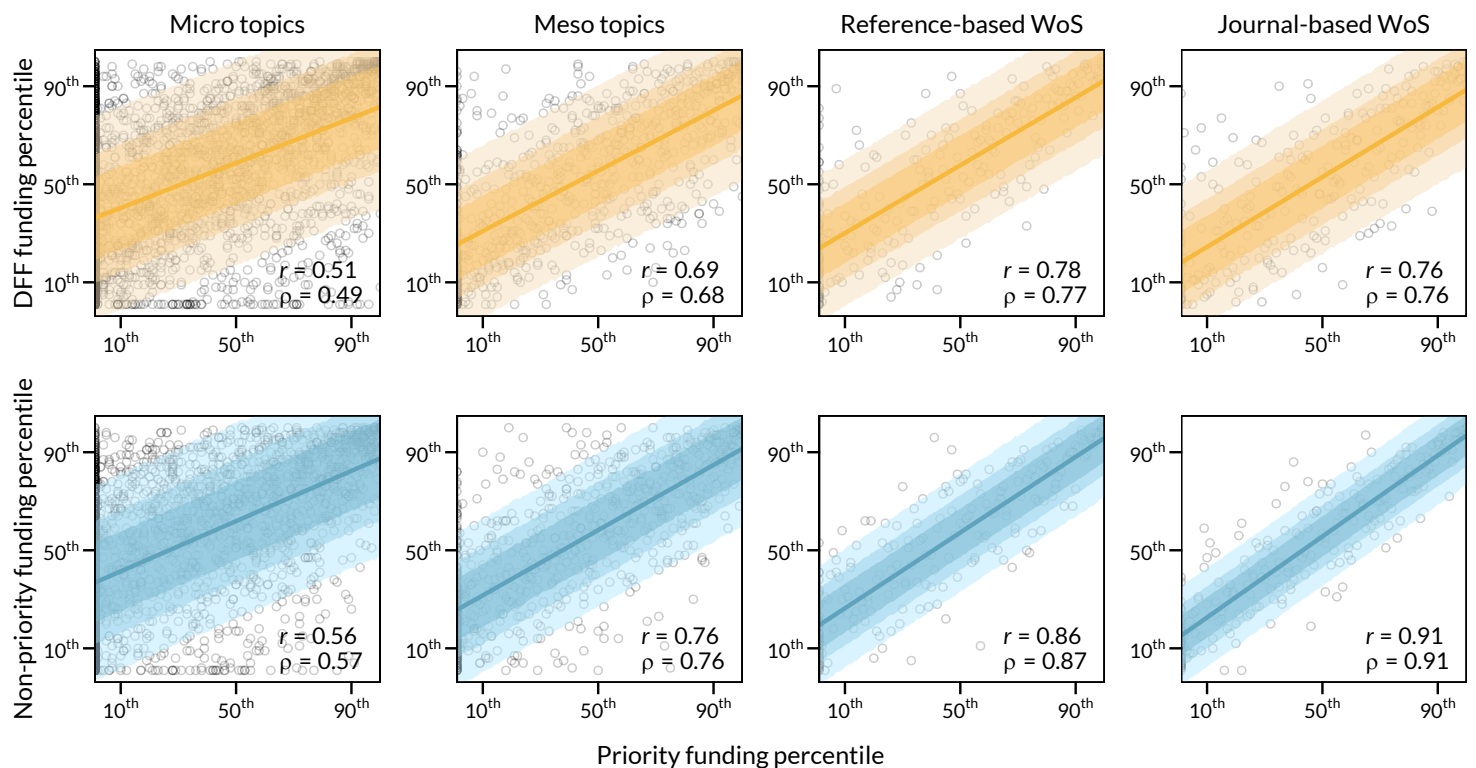

Figure 4: Topical overlap of funded strategic and "free" research in Denmark and the UK. Note: Solid lines are regression fits from a Bayesian linear model, with $50 \%, 70 \%$ and $90 \%$ credible intervals calculated from the posterior predictive distribution. $r=$ Pearson's correlation and $\rho=$ Spearman's rank correlation.

relative priority given by traditional councils and instruments. Instead of comparing the sum of funding for each topic from different sources, I calculate each topic's funding rank for each source and investigate whether the same topic is highly prioritised in each domain. The figure shows the basic scatter plot of funding percentiles. On top of the raw data points, a Bayesian linear regression indicates the degree of correlation between funding percentiles in strategic vs. "free" research councils (Denmark), and priorities vs. non-priorities grants in the EPSRC and BBSRC (UK). The shaded areas are $50 \%, 70 \%$ and $90 \%$ credible intervals calculated from the posterior predictive distribution. ${ }^{5}$

At the systemic level, the correlation between funding priorities in strategic vs. non-strategic funding councils and funding instruments informs us about the overlap in what topics and disciplines are valued (or not). This is of course an abstraction, which factors away the variation in how much money is invested in different types of research by different funders, and focuses on the overlap in priorities for the average topic or discipline. However, the models show that at the disciplinary level, priorities line up to a surprising degree. In both Denmark and the UK, the percentile rank of a discipline within targeted and non-targeted funding instruments correlate positively, with correlation coefficients between 0.76 and 0.91 . The strongest bivariate relationship is between prioritised and non-prioritised grants in the UK, where a one percentile increase in funding from targeted sources leads to a 0.83 increase in percentile rank among non-prioritised sources (based on the original WoS categorisation). In the lower end of

\footnotetext{
${ }^{5}$ See Section B in the supplementary material for a full outline of the modelling procedure.
} 
the spectrum this increase is only 0.69 in the Danish case (reference-based disciplines). With the model, we can use a discipline's funding rank within prioritised instruments to guess its average rank in non-prioritised grant competitions. The simplified model predicts that $50 \%$ of disciplines in the 25th prioritised percentile will fall somewhere between the 28th and 53rd percentile for reference-based disciplines in Denmark, and $50 \%$ from the 75 th percentile will have a non-prioritised rank of 63 to 87 . We should note that while the relationship seems fairly strong at the average, the prediction intervals incorporate all residual variance (not predicted) in a discipline's rank, and highlights that it is likely determined by many other unmeasured factors. At the topical level, the average relationship is weaker and much more uncertain. For meso topics, a 1 percentile rank increase from targeted funding translates into a 0.61 (Denmark) or 0.66 percentile (UK) rank change in non-targeted funding, but here the predicted funding ranks are very uncertain. For the Danish case, a topic in the middle of the targeted funding distribution would have a 50\% probability of attaining a non-targeted rank of 41 to $69.70 \%$ of predictions for an average topic at the middle places it in a percentile rank between 34 and 77. In the UK, the predicted rank interval is almost identical (70 \% fall between 39 and 78 ), and for micro topics the band is even wider. So although there seems to be some overlap at the topical level, it is much more variant than for disciplines. One reason for the large uncertainty for many of the predicted percentile ranks comes from large differences between broad research areas. Section $C$ in the supplementary material reports results from a model similar to figure 4, but with a hierarchical structure estimating separate predictions for five main fields (Biomedical and health sciences, Life and earth sciences, Mathematics and computer science, Physical sciences and engineering, and Social sciences and humanities). For e.g. referencebased disciplines in Denmark, a percentile rank change in targeted funding leads to a average change of 0.76 percentiles for the biomedical fields, but only 0.67 in physics and engineering. These differences are even more pronounced for micro topics, where the corresponding model coefficients are 0.59 in biomedical fields, 0.42 in physics, and only 0.25 in mathematics and computer science. These large field differences stem from the fact that many targeted programmes are implemented within certain parts of the natural and medical sciences.

The question is how the overlap in priorities impact researchers' problem choice. As shown in figure 3, researchers tend to change their topical focus rather infrequently. In figure 5, I show the median similarity between a researchers grant based on targeted funding (represented at year 0 ) and grants obtained before and after this grant in the United Kingdom sample. The figure shows a lot of variation in similarity both before and after receiving a grant with a thematic priority. Similarities are consistently high at the disciplinary level, with scores between 0.7 and 0.8 , while topical similarities tend to fluctuate more. For meso-level topics, the similarity is lowest one year before (0.399) and largest four years after (0.771). Interestingly, both topical and disciplinary similarities to a targeted grant tend to be highest 3-4 years before and after. This coincides with a median grant duration of 2.9 years, and may indicate that grants building on prioritised grant lead to a certain topic lock-in, but PIs are probably running multiple research lines in parallel. 


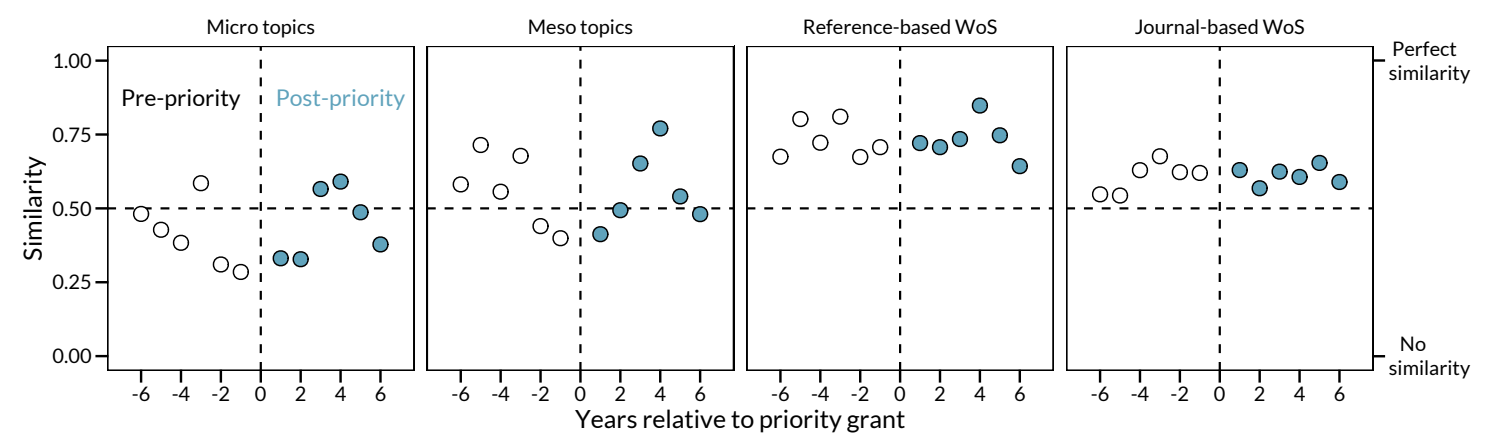

Figure 5: Topical similarity of grants for PIs with targeted funding. Note: Points are median similarity scores relative to a priority grant at year 0 .
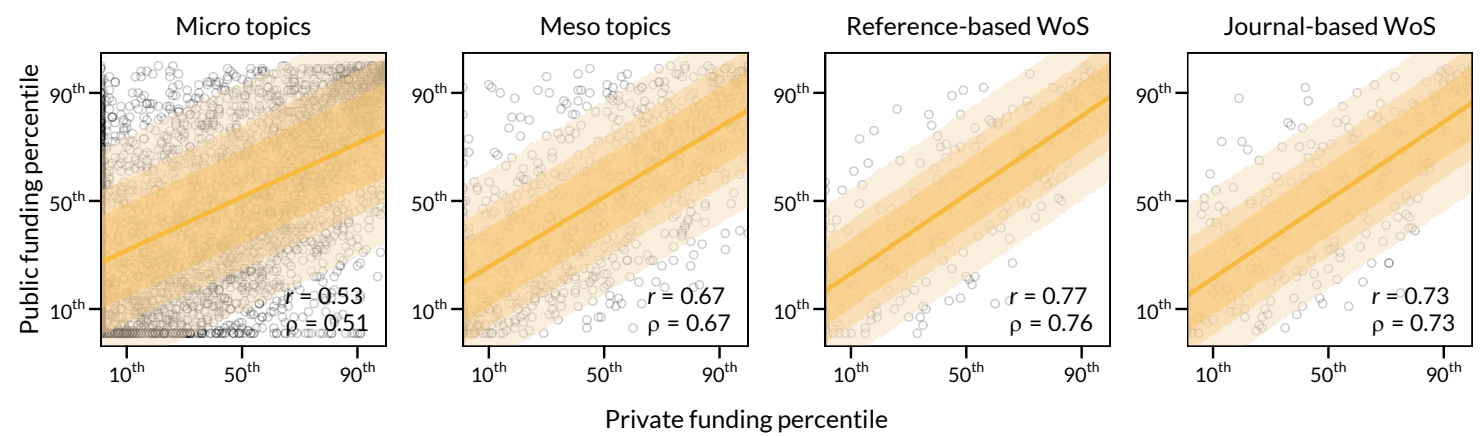

Figure 6: Topical overlap of funded private vs. public research in Denmark.

Note: Solid lines are regression fits from a Bayesian linear model, with $50 \%, 70 \%$ and $90 \%$ credible intervals calculated from the posterior predictive distribution. $r=$ Pearson's correlation and $\rho=$ Spearman's rank correlation.

Priority overlap between public and private funders. In addition to thematic priorities from public funders, there is a growing concern that the increase in funding from private funders contribute to a narrowing of funded topics and disciplines (Whitley et al., 2018; Yegros-Yegros et al., 2020). Below, I use information on grants from all the major public and private research foundations in Denmark to asses the overlap in funded topics. Figure 6 reports bivariate evidence similar to 4 , but swaps funding percentiles from targeted sources to percentiles from private sources.

The overall pattern is similar to the one observed above. The correlation between funding percentiles are strongest for disciplines and lowest for the most detailed topics. As evident by spread of individual topics and disciplines, the prediction of public funding based on private funding is also more uncertain as the categorisation of grants become more granular. For the reference-based disciplines, a one percentile score increase from private sources coincide with a 0.73 percentile increase in public funding. However, the prediction intervals reflect large individual variation for these disciplines. At the 25 th private funding percentile, $50 \%$ of the predicted public funding percentile lies within the [22nd - 47th] interval. Likewise, for disciplines within the 75 th percentile of private funding, the model predicts a public funding 

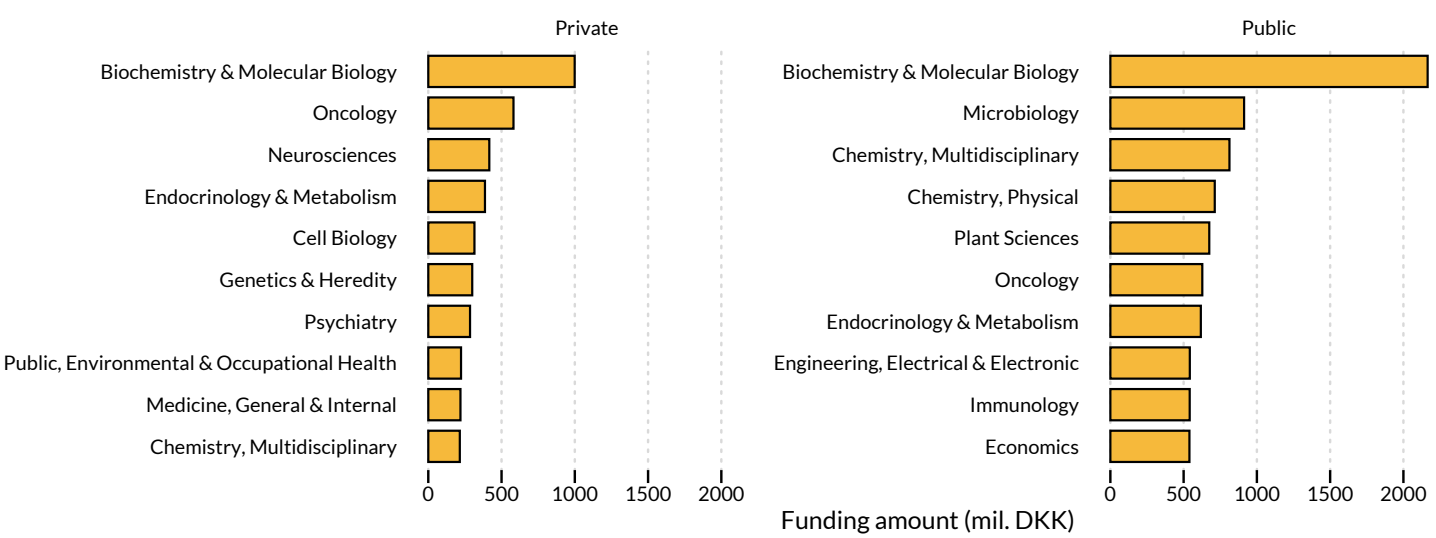

Figure 7: Amount of funding to reference-based disciplinary categories in Denmark.

percentile of 58 to 83 . At the topical level, the regression coefficient for meso topics is 0.64 (95\% credible interval $=0.59-0.70)$ and 0.5 (95\% credible interval $=0.47-0.52)$ for micro topics. However, due to the greater variability for individual topics, the predicted public funding percentiles are more uncertain. For meso topics, \% of predicted percentiles of a topic within the 50th private percentile are between 37 and 67, and for micro topics the range is 34 to 69 . In the case of the Danish private funders, this lack of certainty stems from the funders strong focus on biomedical research. A number of the private research foundations are tied to pharmaceutical companies and invest the bulk of their funding budget in medicine, microbiology, and related fields. For the overlap between private and public funding, I also estimated a model which takes into account differences between the five major research fields (see section $\mathrm{C}$ in the supplementary material). In the domain of micro topics, the 1 private percentile increase is associated with an average 0.75 public percentile increase for the biomedical field compared to only 0.31 for the social sciences and humanities. Looking at the absolute amount of funding for different disciplines confirm the that private and public funders share at least some of their strongly prioritised areas. Figure 7 lists the top 10 funded disciplinary categories, and show that areas such as Biochemistry \& Molecular biology, Cell biology, Micro biology and Endocrinology \& Metabolism are high priority areas for both private and public funders.

\section{Discussion}

The results presented here provide an early empirical exploration of the role of funder priorities in skewing research towards a narrow set of scientific disciplines and topics. Data from both Danish and United Kingdom research funders confirm that competitively awarded grants tend to be accrued by few successful researchers, while the majority of grantees accumulate one to two grants over a prolonged period. Consistent with other studies of research funding (Madsen \& Aagaard, 2020; Stoeger et al., 2018), the same degree of concentration exists for the type of research that is conducted within these grants. Funding is extremely skewed at the disciplinary level, but also for more fine grained topics or problems. The question is whether 
this dual concentration is somehow detrimental to the scientific ecosystem. Striking the right balance between concentration and dispersal of funding is a question of degree, not either or. Researchers generally agree that some measure of concentration is desirable (Aagaard et al., 2020). Not all research is of equal quality, not all problems merit the same level of attention, and some disciplines are naturally more resource dependent. A greater diversity of people and problems cannot become the end goal in itself as it may dilute all concerns for research quality and negate the need for prioritising scarce resources. However, the analysis above raises a number strong empirical indications of a system that has tipped too much in favor of concentration.

First, the analysis shows that the strong concentration on few individuals tend to contribute to less topical diversity because well-funded PIs selectively engage in the most prestigious and highly funded topics. A highly stratified distribution of funding is not in itself a problem, if funding is concentrated on a broader set of disciplines and result in research across a broad spectrum of problems. However, when funding organisations focus on individual applications, and delegate quality assessments to a peer review system, we run the risk of having multiple independent funding decisions skew funding in the aggregate. One problem might be that basing funding allocation on strict quality criteria, including past research performance, neglects a broader perspective on the whole funding portfolio. Individual review panels have little overview of what is funded in other panels or application rounds, meaning that high performing researchers continue to prevail. Different strategies for mitigating this lack of overview have been proposed. Directors at the NIH have proposed a funding cap for individual investigators, of no more than three R01 grants at one time. This policy would supposedly free up funds for 1600 new grants. However, the policy was viewed with strong skepticism by many highly productive PIs and labs, and was subsequently abandoned. We could instead envision less invasive policies for highlighting and monitoring biases connected to a lack of oversight. In order to encourage topical diversity, more funding could be directed towards e.g. Discipline Hopping Awards or grant instruments aimed a building capacity in areas a PI has not explored earlier. This seems pertinent given that researchers tend not to change topics very often, and would likely need large funding incentives to do so (Myers, 2020). Grant proposals should also highlight how a project differs from earlier funded projects, review processes could be completely blinded, or funding could be allocated through a lottery of proposals deemed of sufficient quality (Fang \& Casadevall, 2016). A broader portfolio of grant instruments with different aims and notions of quality will be necessary to broaden the type of research funded.

Second, priorities from targeted funding instruments appear similar to what is funded through regular funding instruments. It is likely that funders have the prestige and perceived opportunity of different research topics and areas in mind when they set specific priorities, and priorities are also set with input from the research community. As a result priorities may be suggested by successful and influential researchers, and/or reviewed and evaluated in councils and working groups where such researchers participate. This reflects a strong power dynamic where a select group of decision makers influence what type of research is deemed a priority. Moreover, because topic choice is hard to influence through priorities (Myers, 2020), the most effective instrument is rather funders' decisions not to fund certain topics (Madsen \& Aagaard, 
2020). When thematic and non-thematic funding instruments overlap this becomes a vicious cycle. If funders had better overviews of what is funded across instruments and individual research councils, charities, and research foundations, they would have an easier time targeting areas with too little support.

Third, private funders will naturally focus their funding allocations. When excellence becomes an overarching quality criteria across the system, topics with little market potential are at a disadvantage. Funding from private sources spill over in publicly funded research because it bolsters the amount of resources available to high performing groups. The results presented here at least hints at this type of spill-over as the funded topics and disciplines across funding sectors coincide. Other research has also shown that the most successful researchers often attract funding from diverse sources, and support their research with both public and private funds (Madsen \& Aagaard, 2020). Research strains with success in private funding will then inadvertently become successful in public funding competition. To counter this, we need greater coordination between funders. This could be through shared priority setting that ensures some degree of functional division of labor between research funding bodies, and continuous coordination in e.g. a national research funding forum.

From my analysis, it is impossible to argue for any causal link from individual concentration to topic concentration, or from thematic priorities to topic concentration. Or rather, we could credibly argue that the connection runs in both directions. Given the complexity of the interlinked and interdependent mechanisms at work, it is likely that funding concentration and research priorities are prone to mutually reinforcing cycles. This does not necessarily invalidate the results of the analysis. The great degree of overlap mandates more research. Future studies could investigate how researchers change topic along with priorities and prestige. Here, we would need in-depth case studies to tease apart the causal mechanism at work, especially to take into account the timing of different events.

\section{References}

Aagaard, K. (2017). The Evolution of a National Research Funding System: Transformative Change Through Layering and Displacement. Minerva, 55(3), 279-297. https:// doi. org/10.1007/s11024-017-9317-1

Aagaard, K., Kladakis, A., \& Nielsen, M. W. (2020). Concentration or dispersal of research funding? Quantitative Science Studies, 1(1), 1-33. https://doi.org/10.1162/qss

Alberts, B., Kirschner, M. W., Tilghman, S., \& Varmus, H. (2014). Rescuing US biomedical research from its systemic flaws. Proceedings of the National Academy of Sciences, 111(16), 5773-5777. https://doi.org/10.1073/pnas.1404402111

Allison, P. D., Long, J. S., \& Krauze, T. K. (1982). Cumulative Advantage and Inequality in Science. American Sociological Review, 47(5), 615-625.

BBSRC. (2020). Responsive mode priorities. https://bbsrc.ukri.org/funding/grants/priorities/

Berg, J. M. (2012). Well-funded investigators should receive extra scrutiny. Nature, 489(7415), 203. https://doi.org/https://doi.org/10.1038/489203a 
Bloch, C., \& Sørensen, M. P. (2015). The size of research funding: Trends and implications. Science and Public Policy, 42(1), 30-43. https://doi.org/10.1093/scipol/scu019

Bol, T., de Vaan, M., \& van de Rijt, A. (2018). The Matthew effect in science funding. Proceedings of the National Academy of Sciences, 1-4. https://doi.org/10.1073/pnas.1719557115

Bürkner, P.-C. (2017). brms : An R Package for Bayesian Multilevel Models. Journal of Statistical Software, 80(1), 1-28. https://doi.org/10.18637/jss.v080.i01

Bürkner, P.-C. (2018). Advanced Bayesian Multilevel Modeling with the R Package brms. The R Journal, 10(1), 395-411.

Ciarli, T., \& Ràfols, I. (2019). The relation between research priorities and societal demands: The case of rice. Research Policy, 48(4), 949-967. https:// doi.org/10.1016/j.respol. 2018.10.027

Cole, S. (1970). Professional Standing and the Reception of Scientific Discoveries. American Journal of Sociology, 76(2), 286-306.

Edwards, A. M., Isserlin, R., Bader, G. D., Frye, S. V., Wilson, T. M., \& Yu, F. H. (2011). Too many roads not taken. Science, 470, 163-165.

Evans, J. A., Shim, J. M., \& Ioannidis, J. P. (2014). Attention to local health burden and the global disparity of health research. PLoS ONE, 9(4), 1-9. https:// doi.org/10.1371/journal. pone.0090147

Fang, F. C., \& Casadevall, A. (2016). Research Funding: the Case for a Modified Lottery. mBio, 7(2), e00694-16. https://doi.org/10.1128/mBio.00694-16

Fortin, J. M., \& Currie, D. J. (2013). Big Science vs. Little Science: How Scientific Impact Scales with Funding. PLoS ONE, 8(6). https://doi.org/10.1371/journal.pone.0065263

Gillum, L. A., Gouveia, C., Dorsey, E. R., Pletcher, M., Mathers, C. D., Charles, E., \& Johnston, S. C. (2011). NIH Disease Funding Levels and Burden of Disease. PLoS ONE, 6(2), e16837. https://doi.org/10.1371/journal.pone.0016837

Gläser, J., \& Laudel, G. (2016). Governing Science. European Journal of Sociology, 57(01), 117168. https://doi.org/10.1017/S0003975616000047

Gläser, J., \& Velarde, K. S. (2018). Changing Funding Arrangements and the Production of Scientific Knowledge: Introduction to the Special. Minerva, 56(1), 1-10. https://doi.org/ 10.1007/s11024-018-9344-6

Gross, C., Anderson, G., \& Powe, N. (1999). The Relation between Funding by the National Institutes of Health and the Burden of Disease. New England Journal of Medicine, 340(24), 1881-1887. https://doi.org/10.1056/NEJM199906173402406

Hellström, T., Jacob, M., \& Sjöö, K. (2017). From thematic to organizational prioritization: The challenges of implementing RDI priorities. Science and Public Policy, 44(5), 599-608. https://doi.org/10.1093/scipol/scw087

Horlings, E., \& Gurney, T. (2013). Search strategies along the academic lifecycle. Scientometrics, 94(3), 1137-1160. https://doi.org/10.1007/s11192-012-0789-3

Jones, R., \& Wilsdon, J. (2018). The Biomedical Bubble: Why UK research and innovation needs a greater diversity of priorities, politics, places and people. London, Nesta. 
Katz, Y., \& Matter, U. (2019). Metrics of Inequality: The Concentration of Resources in the U .S. Biomedical Elite. Science as Culture, O(0), 1-28. https://doi.org/10.1080/09505431. 2019.1694882

Kay, M. (2019). tidybayes: Tidy Data and Geoms for Bayesian Models. https:// doi.org/10. $5281 /$ zenodo.1308151

Kearnes, M., \& Wienroth, M. (2011). Tools of the Trade: UK Research Intermediaries and the Politics of Impacts. Minerva, 49(2), 153-174. https:// doi.org/10.1007/s11024-0119172-4

Laudel, G., \& Gläser, J. (2014). Beyond breakthrough research: Epistemic properties of research and their consequences for research funding. Research Policy, 43(7), 1204-1216. https: //doi.org/10.1016/j.respol.2014.02.006

Ma, A., Mondragón, R. J., \& Latora, V. (2015). Anatomy of funded research in science. Proceedings of the National Academy of Sciences, 112(48), 14760-14765. https:// doi.org/ 10. 1073/pnas. 1513651112

Madsen, E. B., \& Aagaard, K. (2020). Concentration of Danish research funding on individual researchers and research topics: Patterns and potential drivers. Quantitative Science Studies, 1(3), 1159-1181.

Merton, R. K. (1968). The Matthew Effect in Science. Science, 159(3810), 56-63.

Milojević, S. (2020). Practical method to reclassify Web of Science articles into unique subject categories and broad disciplines. Quantitative Science Studies, 1(1), 183-206. https:// doi.org/10.1162/qss

Mongeon, P., Brodeur, C., Beaudry, C., \& Lariviere, V. (2016). Concentration of research funding leads to decreasing marginal returns. Research Evaluation, 25(4), arXiv 1602.07396, 396-404. https://doi.org/10.1093/reseval/rvw007

Myers, K. (2020). The Elasticity of Science. American Economic Journal: Applied Economics, 12(4), 1-38. https://www.ssrn.com/abstract $=3176991$

Nature. (2003). Editorial: Research funding: The problem with priorities. Nature Materials, 2(10), 639. https://doi.org/10.1038/nmat992

Peifer, M. (2017). The argument for diversifying the NIH grant portfolio. Molecular Biology of the Cell, 28(22), 2935-2940. https://doi.org/10.1091/mbc.E17-07-0462

Polanyi, M. (1962). The Republic of Science: Its Political and Economic Theory. Minerva, 1(1), $54-73$.

Rafols, I., Hopkins, M. M., Hoekman, J., Siepel, J., O’Hare, A., Perianes-Rodríguez, A., \& Nightingale, P. (2014). Big Pharma, little science?. A bibliometric perspective on Big Pharma's R\&D decline. Technological Forecasting and Social Change, 81(1), arXiv 1306.0947, 2238. https://doi.org/10.1016/j.techfore.2012.06.007

Røttingen, J.-A., Regmi, S., Eide, M., Young, A. J., Viergever, R. F., Årdal, C., Guzman, J., Edwards, D., Matlin, S. A., \& Terry, R. F. (2013). Mapping of available health research and development data: what's there, what's missing, and what role is there for a global observatory? The Lancet, 382(9900), 1286-1307. https:// doi.org/10.1016/S0140 6736(13)61046-6 
Rzhetsky, A., Foster, J. G., Foster, I. T., \& Evans, J. A. (2015). Choosing experiments to accelerate collective discovery. Proceedings of the National Academy of Sciences, 112(47), arXiv arXiv:1408.1149, 14569-14574. https://doi.org/10.1073/pnas.1509757112

Sarewitz, D., \& Pielke, R. A. (2007). The neglected heart of science policy: reconciling supply of and demand for science. Environmental Science and Policy, 10(1), 5-16. https://doi.org/ 10.1016/j.envsci.2006.10.001

Sjögårde, P., \& Ahlgren, P. (2020). Granularity of algorithmically constructed publication-level classifications of research publications: Identification of specialties. Quantitative Science Studies, 1(1), 207-238. https://doi.org/10.1162/qss

Stewart, J. (1995). Models of priority-setting for public sector research. Research Policy, 24(1), 115-126. https://doi.org/10.1016/0048-7333(93)00752-F

Stoeger, T., Gerlach, M., Morimoto, R. I., \& Nunes Amaral, L. A. (2018). Large-scale investigation of the reasons why potentially important genes are ignored. PLoS Biology, 16(9), 1-25. https://doi.org/10.1371/journal.pbio.2006643

Traag, V. A., Waltman, L., \& Eck, N. J. V. (2019). From Louvain to Leiden : guaranteeing wellconnected communities. Scientific Reports, 9(5233), 1-12. https:// doi.org/10.1038/ s41598-019-41695-z

Wallace, M. L., \& Ràfols, I. (2018). Institutional shaping of research priorities: A case study on avian influenza. Research Policy, 47(10), 1975-1989. https://doi.org/10.1016/j.respol. 2018.07.005

Waltman, L., Rafols, I., Eck, N. J. V., \& Yegros, A. (2019). Supporting priority setting in science using research funding landscapes (No. 1), Research on Research Institute. https://doi.org/10. 6084/m9.figshare.9917825.v1

Waltman, L., \& Van Eck, N.J. (2013). A smart local moving algorithm for large-scale modularitybased community detection. European Physical Journal B, 86(11), arXiv 1308.6604, 471. https://doi.org/10.1140/epjb/e2013-40829-0

Waltman, L., \& van Eck, N. (2012). A New Methodology for Constructing a Publication-Level Classification System of Science. Journal of the American Society for Information Science and Technology, 63(12), arXiv 0803.1716, 2378-2396. https://doi.org/10.1002/asi

Whitley, R., Gläser, J., \& Laudel, G. (2018). The Impact of Changing Funding and Authority Relationships on Scientific Innovations. Minerva, 56(1), 109-134. https://doi.org/10. 1007/s11024-018-9343-7

Wickham, H. (2016). ggplot2: Elegant Graphics for Data Analysis. New York, NY, Springer-Verlag. https://ggplot2.tidyverse.org

Yao, L., Li, Y., Ghosh, S., Evans, J. A., \& Rzhetsky, A. (2015). Health ROI as a measure of misalignment of biomedical needs and resources. Nature Biotechnology, 33(8), 807-811. https: //doi.org/10.1038/nbt.3276

Yegros-Yegros, A., Klippe, W. V. D., Abad-garcia, M. F., \& Rafols, I. (2020). Exploring why global health needs are unmet by research efforts: the potential influences of geography , industry and publication incentives. Health Research Policy and Systems, 18(47), 1-14. 
Zeng, A., Shen, Z., Zhou, J., Fan, Y., Di, Z., Wang, Y., Stanley, H. E., \& Havlin, S. (2019). Increasing trend of scientists to switch between topics. Nature Communications, 10(3439), arXiv arXiv:1812.03643v1, 1-11. https://doi.org/10.1038/s41467-019-11401-8 


\section{Supplementary material}

\section{The Interdependence of Research Funding Concentration, Policy \\ Priorities, and Problem Choice}

July 15, 2020

\section{A. Comparison of grant samples}

Table S1: Summary of grant samples for full and publication-matched samples

\begin{tabular}{lccccc}
\hline & \multicolumn{2}{c}{ Denmark } & & \multicolumn{2}{c}{ United Kingdom } \\
\cline { 2 - 3 } \cline { 5 - 6 } & Full sample & WoS-matched sample & & Full sample & WoS-matched sample \\
\hline No. of grants & 18,404 & 11,605 & & $41,416.0$ & $30,662.0$ \\
No. of PIs & 8,624 & 4,703 & & $22,290.0$ & $14,465.0$ \\
Mean amount & $2,555,962$ & $2,731,366$ & & $434,732.0$ & $586,523.5$ \\
Median amount & 984,191 & $1,000,000$ & & $239,807.2$ & $333,547.9$ \\
Std. dev. of amount $^{\mathrm{a}}$ & $6,690,068$ & $7,103,392$ & & $1,425,052.0$ & $1,745,664.5$ \\
\hline
\end{tabular}

Note: ${ }^{\text {a }}$ Values are given in DKK and CPI-adjusted pounds respectively. 


\section{B. Formal definitions of models for priority overlap}

To assess the degree of overlap in priorities between both targeted vs. non-targeted and private vs. public funding, I use regression models to quantify both the average degree of overlap, the corresponding uncertainty in this average, and the remaining variation in the data. For figure 4 and 6, the models are simple linear regressions, while supplementary analyses are hierarchical linear models. The formal definitions of these models follow here.

Simple overall models. Let $y$ denote the calculated funding percentile in non-targeted funding instruments (for figure 4) or public research councils (for figure 6), and $i$ the index for individual topics (micro or meso) or discipline (reference-based or journal-based). Then:

$$
y_{i} \sim \operatorname{Normal}\left(\mu_{i}, \sigma\right)
$$

The expression for $\mu_{i}$ specify the average funding percentile in non-targeted/public funding as a linear function of the funding percentile in targeted/private instruments for all topics or disciplines $i$ :

$$
\mu_{i}=\alpha_{0}+\beta_{1} P_{i}
$$

The parameter $\beta_{i}$ then represents the average difference in funding percentile between an average topic and an average topic with a one point higher funding percentile. To recover posterior distributions of the parameters $\alpha_{0}, \beta_{1}$, and $\sigma$, we define their distributions through the following priors:

$$
\begin{gathered}
\alpha_{0} \sim \operatorname{Normal}(50,20) \\
\beta_{1} \sim \operatorname{Normal}(0.5,1) \\
\sigma \sim \operatorname{Half-normal}(0,20)
\end{gathered}
$$

These priors allow for funding percentile overlap to be both negatively and positively related, with $95 \%$ of parameter values between -1.48 and 2.42 (see coloured area in figure S1). Because Madsen and Aagaard (2020) find a positive relationship between funding percentiles between public and private funders for disease related research, the prior on $\beta_{1}$ puts around $70 \%$ of probability mass above zero, and it thus induces some skepticism towards negative relationships between funding percentiles.

Random effects on research area. Because thematic priorities are often applied unequally across research areas, and private funders in Denmark are focused on the biomedical and natural sciences, I account for this difference by estimating a hierarchical model, where the degree of overlap in percentiles, i.e. $\beta_{1}$ and $\alpha_{0}$, can vary between the five research areas. The model builds on the basic bivariate model from above, but adds some parameters: 


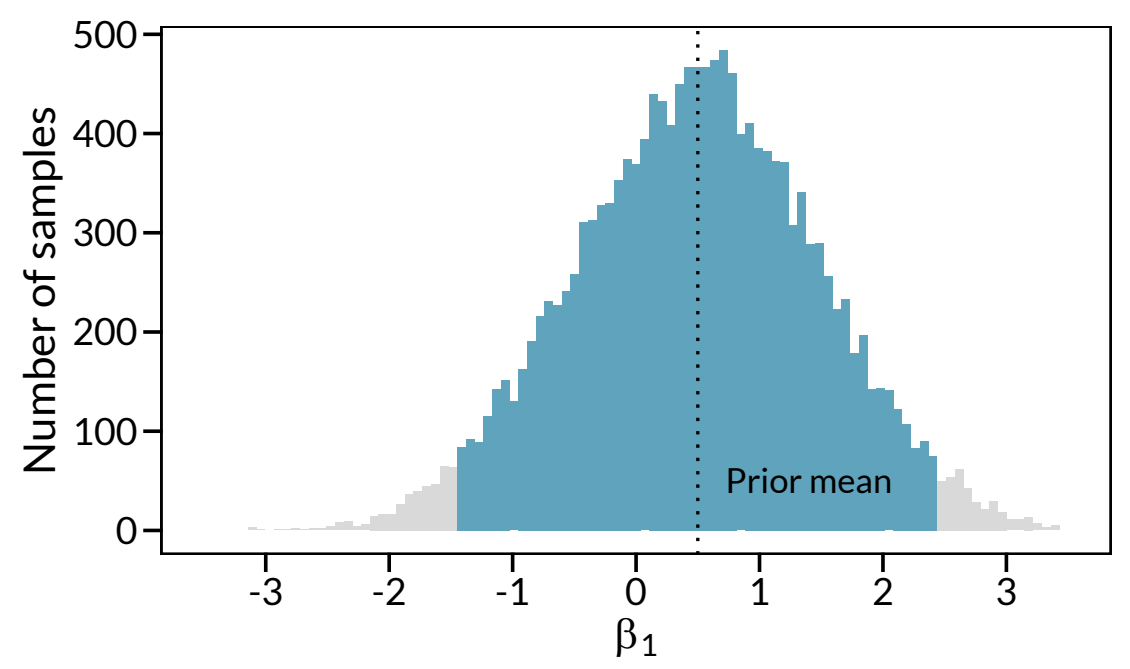

Figure S1: Prior distribution for priority overlap $\left(\beta_{1}\right)$.

$$
\begin{gathered}
y_{i} \sim \operatorname{Normal}\left(\mu_{i}, \sigma\right) \\
\mu_{i}=\alpha_{0}+\alpha_{j}+\left(\beta_{0}+\beta_{J}\right) P_{i j}
\end{gathered}
$$

Here, $\alpha_{0}$ is the average intercept across five areas $j, \alpha_{j}$ is the varying offsets from the global intercept, $\beta_{0}$ is the average degree of overlap, while $\beta_{j}$ is the offsets from this for each research area $j$. For both $\alpha_{0}$ and $\beta_{0}$, the priors are identical to the ones presented above. I add priors for the standard deviations among intercepts and $\beta$-coefficients, and for the correlation matrix $\mathbf{R}$ of all the varying intercepts and effects:

$$
\begin{gathered}
\sigma_{\alpha} \sim \operatorname{Normal}(0,20) \\
\sigma_{\beta} \sim \operatorname{Normal}(0,1) \\
\mathbf{R} \sim \operatorname{LKJ}(2)
\end{gathered}
$$

where the LKJ prior on $\mathbf{R}$ is just a weakly informative regularising prior skeptical of extreme correlations.

Estimating the models. All posterior distributions from the models were estimated empirically by Hamiltonian Markov Chain Monte Carlo (HMC) using R (ver. 3.6.3) and the package 'brms' (Bürkner, 2017, 2018). For all models, I assigned 1,000 burn-in samples and 5,000 iterations for the sampler, using four chains, an acceptance rate of 0.99 (adapt delta), and a max tree depth of 15. The visualisations of the posterior distributions and the posterior predictions were made with the R packages 'tidybayes' (Kay, 2019) and 'ggplot2' (Wickham, 2016). 


\section{Results from hierarchical models of priority overlap}

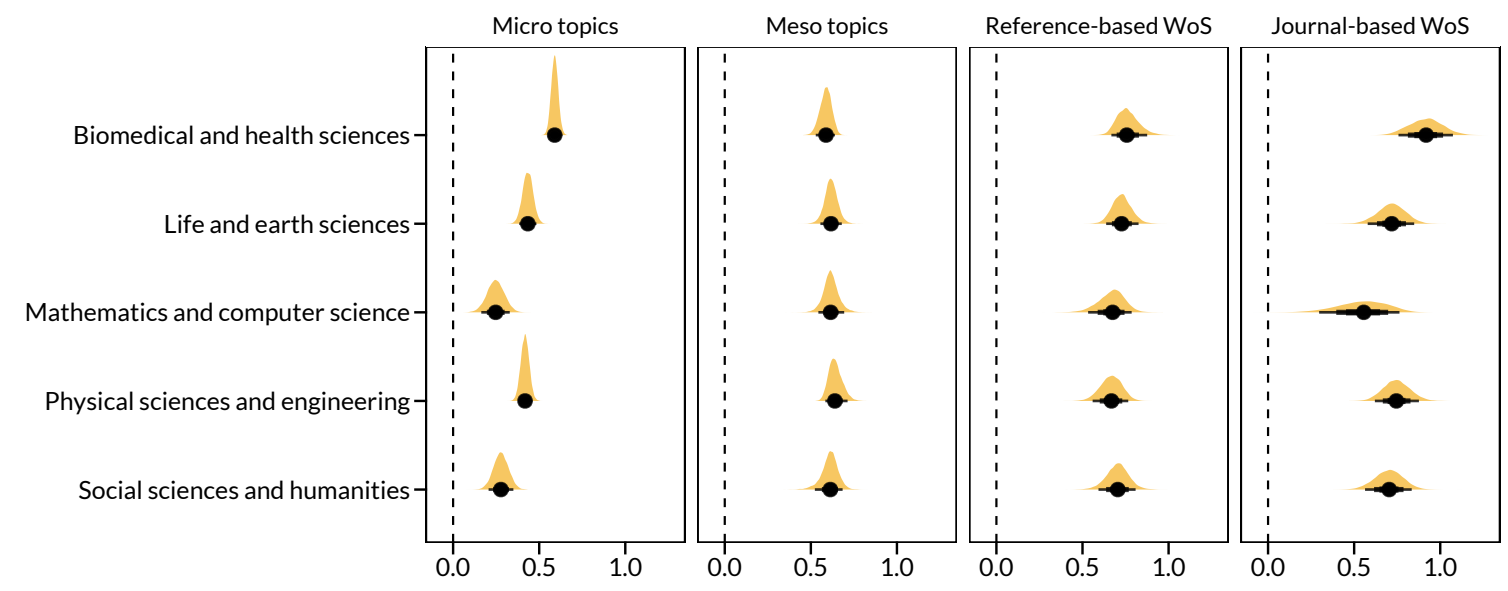

Figure S2: Topical overlap of funded strategic and "free" research in Denmark across five research areas. Note: Points are posterior medians, with $50 \%, 70 \%$ and $90 \%$ credible intervals calculated from the posterior distribution.
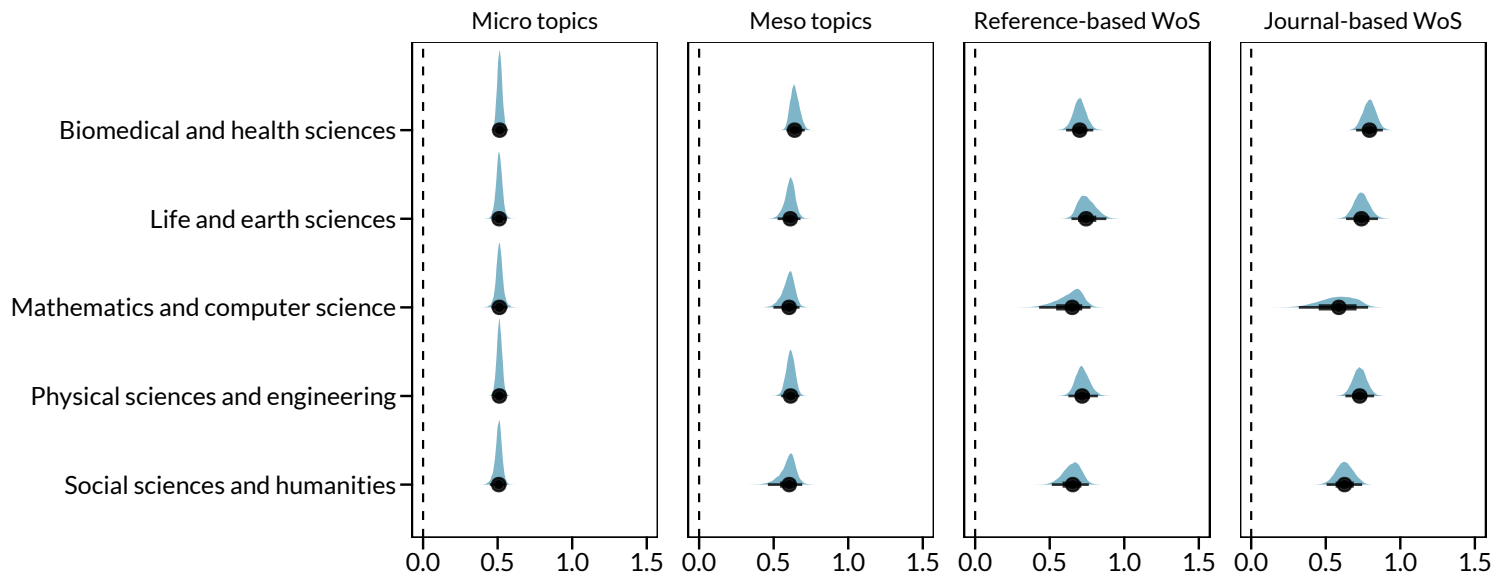

Figure S3: Topical overlap of funded strategic and "free" research in the UK across five research areas. Note: Points are posterior medians, with $50 \%, 70 \%$ and $90 \%$ credible intervals calculated from the posterior distribution. 


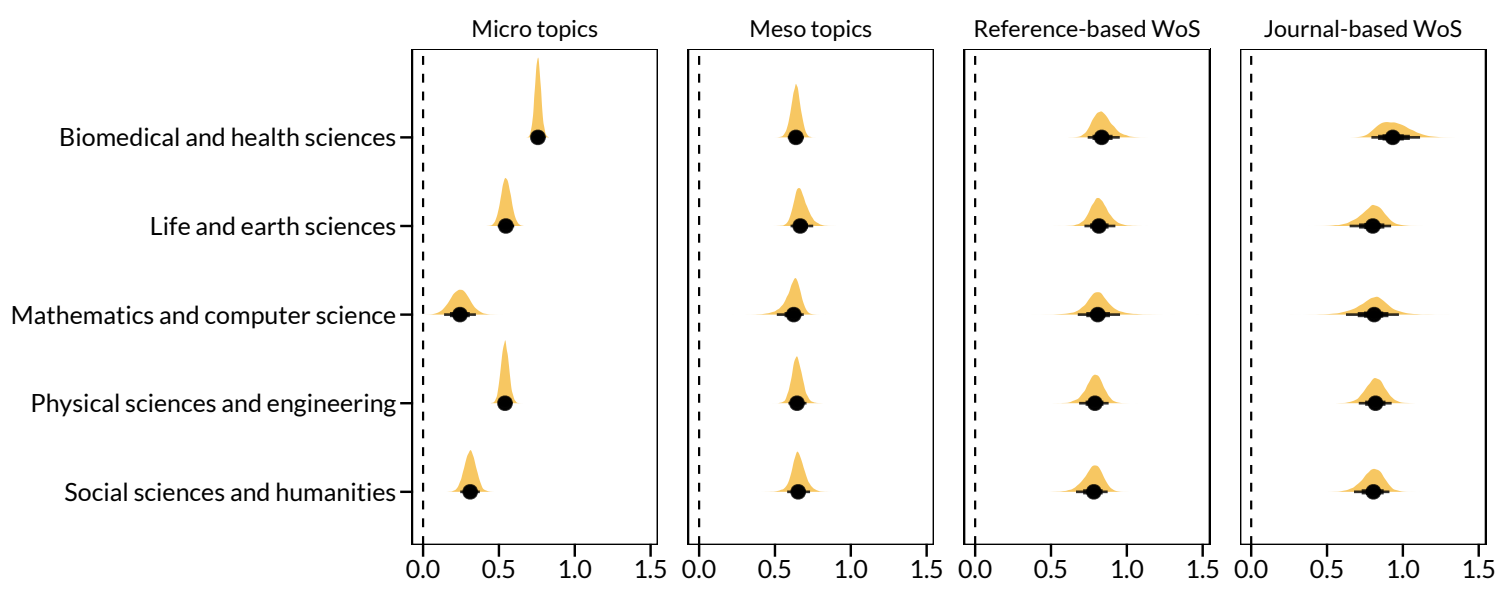

Figure S4: Topical overlap of funded private vs. public research in Denmark across five research areas. Note: Points are posterior medians, with $50 \%, 70 \%$ and $90 \%$ credible intervals calculated from the posterior distribution. 